\title{
EFFECTIVE UTILIZATION OF IDLE TIME IN AN $(s, S)$ INVENTORY WITH POSITIVE SERVICE TIME
}

\author{
A. KRISHNAMOORTHY, T. G. DEEPAK, VISWANATH C. NARAYANAN, AND \\ K. VINEETHA
}

Received 12 December 2005; Revised 18 May 2006; Accepted 18 May 2006

We deviate from the Berman et al. models in inventory with positive service time. We establish a necessary and sufficient condition for system stability. Several performance measures are computed. An optimisation problem is discussed. Our analysis suggests that it is optimal to place the replenishment order when the inventory level is positive (even in the zero lead time case). Numerical illustrations are provided.

Copyright ( 2006 A. Krishnamoorthy et al. This is an open access article distributed under the Creative Commons Attribution License, which permits unrestricted use, distribution, and reproduction in any medium, provided the original work is properly cited.

\section{Introduction}

We consider an $(s, S)$ inventory system with service time with some additional features. To highlight the main additional feature, we first of all describe an inventory with positive service time. Until 1993, inventory models discussed in the literature did not take into consideration the service time; rather it was assumed uniformly that service time is negligible. Hence if the item is available at a demand epoch, it is served instantly. However, there are various situations arising in real life situations where positive processing time is involved before an item is delivered to the customer. The first of such result is due to Berman et al. [1] where they consider deterministic modelling. Berman and Kim [2] deal with stochastic inventory models with positive service time wherein the average cost is minimised using dynamic programming technique. Berman and Sapna [3] consider an $(s, S)$ inventory with positive service time. They consider a finite state model and identify a Markov renewal process to study the system behaviour. In all these models, the processing (service) starts only on the arrival of a customer; in the absence of customers (and of course in the absence of the item (inventory)) the server remains idle.

Now to the present problem. We relax the condition stated in the last sentence of the previous paragraph as follows. If an item is available at a service completion epoch, the 
server processes it (even in the absence of a customer-e.g., assembling of parts); it keeps on doing this as long as unprocessed items are available in the inventory, keeping the sum total of processed and unprocessed items (inclusive of the one being processed, if any) at most $S$ (the maximum that can be held in the inventory). Thus at a customer departure epoch, either there is no processed item available or one or more (a maximum of $S$ ) processed items available. We assume that ours is an $(s, S)$ system. Hence the sum of processed and unprocessed items together cannot exceed $S$. The processing of items even in the absence of customers can ensure reduction in the waiting time and hence in the associated cost. And if there is a queue of customers formed, then no processed item will be available. As long as processed items are available, queue of customers cannot get formed and at an epoch of a customer arrival if a processed item is available, his service time is negligible (like in a classical inventory model); otherwise, he has to wait to get the item served. In a paper to follow, we consider separate stream demands for unprocessed items also.

To describe our problem, we need a three-dimensional representation, in the simplest case. This will be done in the next section. Note that even in the simplest of cases (system remaining Markovian), we will not get analytical solution! Hence we proceed for an algorithmic analysis of the system.

This paper is arranged as follows: next section provides a description of the problem at hand and its mathematical modelling. In the section to follow, we provide the analysis of the model. We obtain the stability condition and the system state probabilities under that regime. Several performance measures are provided in Section 3. In Section 4, we discuss some associated control problem.

\section{Description of the model and its mathematical formulation}

We have a single commodity inventory system operating under the $(s, S)$ policy. Unlike classical inventory model, there is a service time associated with each demand. However, items are serviced (processed) (e.g., assembling) even in the absence of a demand. Thus processed items are stacked separately. The server keeps processing the items. The total of processed and unprocessed items cannot exceed $S$. Also when the total reaches $s$, an order for replenishment is placed and the order materialisation takes place instantly (i.e., lead time is zero).

Demands for the item arrive according to a Poisson process of rate $\lambda$. Service (process) times are exponentially distributed with parameter $\mu$. A queue of customers is formed in the absence of processed items at demand epochs. There is no bound assumed to the queue. Thus the queue of customers can be arbitrarily large. We investigate its stability. The investigation of the maximum number of processed items that may be stacked is also important. This is so since it is much more expensive to stack processed items than unprocessed items. Also note that unlike in classical inventory with zero lead time and inventory with positive service time and zero lead time, we expect a positive reorder level $(s)$ as optimal since this will reduce the waiting time of customers and the consequent cost, thus bringing down the otherwise avoidable holding cost of customers.

Denote by $N(t)$ the number of customers in the systems at time $t$; by $I(t)$ the total inventory (processed plus unprocessed) at $t$; and by $C(t)$ the number of processed items. 
A. Krishnamoorthy et al. 3

Thus $\left\{(N(t), I(t), C(t)), t \in R_{+}\right\}$is a Markov chain on $\{(i, j, k) \mid i=0, S \geq j \geq s+1, j \geq$ $k \geq 0\} \cup\{(i, j, 0) \mid i>0, S \geq j \geq s+1\}$.

Notice that $C(t)$ can be positive only when the number of customers waiting in the system is zero. We investigate the optimal $(s, S)$ values and also the maximum number of processed items that could be stored so that the average system running cost is minimum.

\section{Analysis of the system}

Let $\underline{0}=((0, s+1,0), \ldots,(0, s+1, s+1),(0, s+2,0), \ldots,(0, s+2, s+2), \ldots,(0, S, 0), \ldots,(0, S, S))$ and $\underline{i}=((i, s+1,0),(i, s+2,0)(i, s+2,0), \ldots,(i, S, 0))$, for $i=1,2,3, \ldots$

Then the resulting process is a level independent quasi birth-death process (LIQBD) with the infinitesimal generator

$$
Q=\frac{\underline{1}}{\underline{0}} \underline{\underline{2}}\left(\begin{array}{cccccc}
\underline{0} & \underline{1} & \underline{2} & & & \\
A_{00} & A_{01} & 0 & & & \cdots \\
A_{10} & A_{1} & A_{0} & 0 & \\
0 & A_{2} & A_{1} & A_{0} & & \ldots \\
0 & & A_{2} & A_{1} & A_{0} & \ldots
\end{array}\right),
$$

where

$$
\begin{aligned}
& A_{00}=\left[\begin{array}{cccc}
B_{s+2} & 0 & \cdots & C_{s+1}^{\prime} \\
C_{s+2} & B_{s+3} & \cdots & 0 \\
\vdots & & & \\
0 & 0 & C_{S} & B_{S+1}
\end{array}\right] \\
& A_{01}=\left[\begin{array}{c}
D_{1} \\
D_{2} \\
\vdots \\
D_{S-s}
\end{array}\right] \\
& A_{10}=\left[\begin{array}{cccccccccccccc}
0 & \cdots & 0 & 0 & \cdots & 0 & \cdots & 0 & \cdots & 0 & \mu & 0 & \cdots & 0 \\
\mu & \cdots & 0 & 0 & \cdots & 0 & \cdots & 0 & \cdots & 0 & 0 & 0 & \cdots & 0 \\
0 & \cdots & 0 & \mu & \cdots & 0 & \cdots & 0 & \cdots & 0 & \cdots & \cdots & \cdots & 0 \\
0 & \cdots & 0 & 0 & \cdots & 0 & \cdots & \mu & \cdots & 0 & 0 & \cdots & \cdots & 0
\end{array}\right]
\end{aligned}
$$


4 Utilization of idle time in inventory

is a matrix of order

$$
\begin{aligned}
& (S-s) \times\left((S-s) s+\frac{(S-s)(S-s+1)}{2}+S-s\right), \\
& A_{0}=\lambda I_{S-s}, \\
& A_{1}=-(\lambda+\mu) I_{S-s}, \\
& A_{2}=\left[\begin{array}{ccc}
0 & \cdots & \mu \\
\mu & 0 \cdots & 0 \\
0 & \mu \cdots & 0 \\
0 & \cdots \mu & 0
\end{array}\right]_{(S-s) \times(S-s)}, \\
& B_{j}=\left[\begin{array}{ccccc}
-(\lambda+\mu) & \mu & 0 & \cdots & 0 \\
0 & -(\lambda+\mu) & \mu & 0 \cdots & 0 \\
\vdots & & & & \\
0 & & & -(\lambda+\mu) & \mu \\
0 & & & & -\lambda
\end{array}\right]_{j \times j} \text {, } \\
& C_{j}=\left[\begin{array}{c}
0 \\
\lambda I_{j}
\end{array}\right]_{(j+1) \times j}, \\
& C_{s+1}^{\prime}=\left[\begin{array}{cc}
0 & 0 \\
\lambda I_{s+1} & 0
\end{array}\right]_{(s+2) \times(S+1)}, \\
& D_{1}=\left[\begin{array}{cccc}
\lambda & 0 & \cdots & 0 \\
0 & \cdots & \cdots & 0 \\
0 & \cdots & \cdots & 0
\end{array}\right]_{(s+2) \times(S-s)}, \\
& D_{2}=\left[\begin{array}{ccccc}
0 & \lambda & 0 & \cdots & 0 \\
0 & 0 & \cdots & \cdots & 0 \\
\vdots & & & & \\
0 & \cdots & \cdots & \cdots & 0
\end{array}\right]_{(s+3) \times(S-s)}, \\
& D_{S-s}=\left[\begin{array}{cccc}
0 & \cdots & \cdots & \lambda \\
0 & \cdots & \cdots & 0 \\
0 & \cdots & \cdots & 0
\end{array}\right]_{(S+1) \times(S-s)}
\end{aligned}
$$

3.1. Calculation of steady state probabilities. Let $\pi=\left(\pi_{0}, \pi_{1}, \pi_{2}, \ldots\right)$ be the stationary probability vector associated with $Q$. Note that $\pi_{i}$ is the probability vector associated with level $i$. Then $\pi Q=0$ and $\pi \mathbf{e}=1$, where $\mathbf{e}$ is the column vector of 1's of appropriate dimension. The $\pi_{i}$ are given by

$$
\pi_{i}=\pi_{1} R^{i-1} \quad \text { for } i \geq 2,
$$


where $R$ is the minimal nonnegative solution of the matrix equation $A_{0}+R A_{1}+R^{2} A_{2}=0$. (For details, see Neuts [5].) $\pi_{0}$ and $\pi_{1}$ are calculated from the equations

$$
\begin{gathered}
\pi_{0} A_{00}+\pi_{1} A_{10}=0, \\
\pi_{0} A_{01}+\pi_{1} A_{1}+\pi_{2} A_{2}=0 .
\end{gathered}
$$

Using (3.4), (3.6) can be rewritten as

$$
\pi_{0} A_{01}+\pi_{1}\left(A_{1}+R A_{2}\right)=0 .
$$

From (3.5), we have

$$
\pi_{0}=\pi_{1}\left(-A_{10}\right) A_{00}^{-1} .
$$

Using (3.8) in (3.7), we get

$$
\pi_{1}\left(\left(-A_{10}\right) A_{00}^{-1} A_{01}+A_{1}+R A_{2}\right)=0 .
$$

Thus $\pi_{0}$ and $\pi_{i}(i \geq 2)$ are expressed in terms of $\pi_{1}$, and $\pi_{1}$ can be obtained by solving (3.9) subject to the condition that

$$
\pi_{1}\left(\left(-A_{10}\right) A_{00}^{-1} \mathbf{e}+\mathbf{e}+R(I-R)^{-1} \mathbf{e}\right)=1 .
$$

Let $T=\left(-A_{10}\right) A_{00}^{-1} A_{01}+A_{1}+R A_{2}$. Then (3.9) reads as $\pi_{1} T=0$. Write $T$ as $T=T_{1}-T_{2}$, where $T_{1}=T_{U}+T_{D}$ and $T_{2}=-T_{L}$. Then by block Gauss-Seidel method, the recursive scheme of equations is given by $\pi_{1}^{(l+1)} T_{1}=\pi_{1}^{(l)} T_{2}$.

3.2. Stability criterion. At first glance, it may appear that the stability condition can be weaker than $\lambda<\mu$. Such suspicion arises out of the fact that some customers have zero waiting time. However, it turns out that a few of the items were processed by the server in the absence of the customers and hence such customers who encounter the system with processed items do not have to wait. In any case, service was given and thus a certain amount of time was already spent on processed items towards service. Hence here also we can expect that $\lambda<\mu$, which is true and its proof is given below.

THeOrem 3.1. The system is stable if and only if $\lambda<\mu$.

This follows from the fact that if the rate of drift from level $i$ to $i-1$ is greater than that to level $i+1$ (the two immediate neighbours of $i$ and the process is LIQBD which is skip free to either direction) then $\Pi A_{2} \mathbf{e}>\Pi A_{0} \mathbf{e}$ (see Neuts [5]), where $\Pi$ is the stationary probability vector associated with $A=A_{0}+A_{1}+A_{2}$. This on simplification gives $\lambda<\mu$.

3.3. First passage time analysis. Here we obtain expression for the first passage time probability from a level $i$ to the level $i-1$ for $i \geq 1$. This provides the mean number of customers served during the transition of the number of customers from $i$ to $i-1$. Also it provides the mean time for the above transition. These measures are helpful in the design of service facilities. 
Let $G_{j j}(k, x)$ be the conditional probability that the Markov process, starting in the state $(i, j, 0)$ (for $i>1$ ), at time $t=0$, reaches the level $i-1$ for the first time at or prior to $x$, after exactly $k$ transitions to the left (i.e., after exactly $k$ service completions) and does so by entering the state $\left(i-1, j^{\prime}, 0\right)$ for $s+1 \leq j^{\prime} \leq S$. The matrix with elements $G_{j j^{\prime}}(k, x)$ is denoted by $G(k, x)$. Let $G^{*}(z, \theta)=\sum_{k=1}^{\infty} z^{k} \int_{0}^{\infty} e^{-\theta x} d G(k, x)$.

Then, for $0<z<1, \theta>0$, the matrix $G^{*}(z, \theta)$ is the minimal nonnegative solution to the equation. $z A_{2}\left(\theta I-A_{1}\right) G^{*}(z, \theta)+A_{0} G^{*^{2}}(z, \theta)=0 \lim _{s \rightarrow 0, z \rightarrow 1} G^{*}(z, \theta)=G=\left(G_{j j^{\prime}}\right)$, where

$$
G_{j j^{\prime}}=\operatorname{Pr}\left\{\tau<\infty,\left(N(\tau), M(\tau), M_{1}(\tau)\right)=\left(i-1, j^{\prime}, 0\right) \mid(N(0), M(0), M,(0))=(i, j, 0)\right\}
$$

and $\tau$ is the first passage time from the level $i$ to the level $i-1$.

Let $M_{i j}$ be the mean first passage time from the level $i(i>1)$ to the level $i-1$, given that it started in the state $(i, j, 0)$ and let $\widetilde{M_{1}}$ be a row vector of dimension $S-s$ with elements $M_{1 j}$. Let $M_{2 j}$ be the mean number of service completions during this first passage time and let $\widetilde{M_{2}}$ be a row vector of dimension $S-s$ with elements $M_{2 j}$.

Then

$$
\begin{aligned}
& \widetilde{M_{1}}=-\left.\frac{\partial}{\partial s} G^{*}(z, \theta) e\right|_{\theta=0, z=1}=-\left(A_{1}+A_{0}(I+G)\right)^{-1} e, \\
& \widetilde{M_{2}}=-\left(A_{1}+A_{0}(I+G)\right) A_{2} e\left[\left.\frac{\partial}{\partial z} G^{*}(z, \theta) e\right|_{\theta=0, z=1}\right] .
\end{aligned}
$$

Similar to $G^{*}(z, \theta), \widetilde{M_{1}}$, and $\widetilde{M_{2}}$, we define matrices $G^{*(1,0)}(z, \theta), \widetilde{M}_{1}^{(1,0)}$, and $\widetilde{M}_{2}^{(1,0)}$ for the first passage time from the level 1 to the level 0 and $G^{*(0.0)}(z, \theta), \widetilde{M}_{1}^{(0,0)}$ and $\widetilde{M}_{2}^{(0,0)}$ for the first passage time from the level 0 to the level 0 .

Then $G^{*(1,0)}(z, \theta)=z\left(\theta I-A_{1}\right)^{-1} A_{10}+\left(\theta I-A_{1}\right)^{-1} A_{0} G^{*}(z, \theta) G^{*(1,0)}(z, \theta)$ and $G^{*(0,0)}(z$, $\theta)=\left(\theta I-A_{00}\right)^{-1} A_{01} G^{*(1,0)}(z, \theta)$.

Hence

$$
\begin{aligned}
\widetilde{M}_{1}^{(1,0)} & =-\left.\frac{\partial}{\partial \theta} G^{*(1,0)}(z, \theta) e\right|_{\theta=0, z=1}=-\left(A_{1}+A_{0} G\right)^{-1}\left(A_{0} \widetilde{M}_{1}+e\right), \\
\widetilde{M}_{2} & =\left.\frac{\partial}{\partial z} G^{*(1,0)}(z, \theta) e\right|_{\theta=0, z=1}=-\left(A_{1}+A_{0} G\right)^{-1}\left(A_{0} \widetilde{M}_{2}+A_{10} e\right), \\
\widetilde{M}_{1}^{(0,0)} & =-\left.\frac{\partial}{\partial \theta} G^{*(0,0)}(z, \theta) e\right|_{\theta=0, z=1}=-A_{00}^{-1}\left(A_{01} \widetilde{M}_{1}^{(1,0)}+e\right), \\
\widetilde{M}_{2}^{(0,0)} & =\left.\frac{\partial}{\partial z} G^{*(0,0)}(z, \theta) e\right|_{\theta=0, z=1}=-A_{00}^{-1} A_{01} \widetilde{M}_{2}^{(1,0)} .
\end{aligned}
$$


3.4. Performance measures. (a) Average queue size $=\sum_{i=1}^{\infty} i \pi_{i} \mathbf{e}$

$$
\begin{aligned}
& =\pi_{1} \mathbf{e}+\sum_{i=2}^{\infty} i \pi_{1} R^{i-1} \mathbf{e} \\
& =\pi_{1}\left(I+2 R+3 R^{2}+\cdots\right) \mathbf{e} \\
& =\pi_{1}(I-R)^{-2} \mathbf{e} .
\end{aligned}
$$

(b) If we partition $\pi_{i}$ by states in level $i$ as $\pi_{i}=\left(\pi_{i, s+1,0}, \ldots, \pi_{i, s+1, s+1}, \ldots, \pi_{i S S}\right)$, then the average inventory size (processed + unprocessed) is

$$
\begin{aligned}
& =\sum_{i=0}^{\infty} \sum_{j=s+1}^{S} j \pi_{i j 0}+\sum_{j=s+1}^{S} \sum_{k=1}^{j} j \pi_{0 j k} \\
& =\sum_{i=0}^{\infty} \sum_{j=s+1}^{S} \sum_{k=0}^{j} j \times \pi_{i j k} .
\end{aligned}
$$

(c) Average number of processed items held $=\sum_{j=s+1}^{S} \sum_{k=1}^{j} k \pi_{0 j k}$.

(d) Average service rate $=\lambda \sum_{j=s+1}^{S} \sum_{k=1}^{j} \pi_{0 j k}+\mu \sum_{i=1}^{\infty} \sum_{j=s+1}^{S} \pi_{i j 0}$.

(e) Probability of a customer getting serviced instantaneously (i.e., his service time is negligible, i.e., waiting time is zero) $=\sum_{j=s+1}^{S} \sum_{k=1}^{j} \pi_{0 j k}$.

(f) Probability that a customer will have to wait for service $=\sum_{i=0}^{\infty} \sum_{j=s+1}^{S} \pi_{i j 0}$.

(g) Average replenishment rate $=\lambda \sum_{k=1}^{s+1} \pi_{0, s+1, k}+\mu \sum_{i=1}^{\infty} \pi_{i, s+1,0}$.

(h) $\pi_{0 j j}$ stands for the probability that there is no customer (first subscript) in the system, $j$ items are in the inventory (middle subscript) and all these are in processed state (last subscript). Only when no customer is present, there can be processed inventory. Thus the probability that all these are processed is given by $\sum_{j=s+1}^{S} \pi_{0 j j}$.

(i) Probability that there is no processed item in the inventory $=\sum_{i=0}^{\infty} \sum_{j=s+1}^{S} \pi_{i j 0}$.

(j) Probability that the inventory size is maximum (i.e., $S)=\sum_{i=0}^{\infty} \pi_{i S 0}+\sum_{k=1}^{S} \pi_{0 S k}$.

(k) Average waiting time of an arbitrary customer in the system

$$
=\frac{1}{\mu}\left[\sum_{j=s+1}^{S} \pi_{0 j 0}+\sum_{i=1}^{\infty} \sum_{j=s+1}^{S} i \pi_{i j 0}\right] .
$$

(1) Probability that the inventory level moves from $S$ back to $S$ without any intervening arrivals having to wait

$$
\sum_{i_{1}=1}^{S} \sum_{i_{2}=1}^{S-1} \cdots \sum_{i_{S-s}=1}^{s+1} \pi_{0 S i_{1}} \pi_{0, S-1, i_{2}} \cdots \pi_{0, s+1, i_{S-s}}
$$

(m) Probability that during an $S$ to $S$ transition, all customers have to wait is given by

$$
\sum_{i_{0} \geq 1} \sum_{i_{1} \geq i_{0}-1} \cdots \sum_{i_{S-(s+2)} \geq i_{S-(s+3)}-1} \sum_{i_{S-(s+1)} \geq i_{S-(s+2)}-1} \pi_{i_{0} S 0} \pi_{i_{1}, S-1,0} \cdots \pi_{i_{S-(s+1)}, s+1,0 .} .
$$


The reasoning is as follows: there are $i_{0}(\geq 1)$ customers when a replenishment takes place. At the first departure epoch, there are $i_{1}$ customers left $\left(i_{1} \geq 0\right)$; if this is zero, an arrival should take place before the next service commences, else the next service commences immediately; the $S-(s+1)$ th service in that cycle, with $s+1$ items in the inventory, proceeds and leaves behind one or more customers at its departure and the next service starts. Also the replenishment takes place.

\section{Control problem}

We notice a glaring departure from classical inventory and inventory with service (as introduced by Berman et al.) on the one side and the problem under discussion here on the other side. In the former, the optimal reorder level is zero (whenever lead time is zero) whereas in the latter this is not always true. Here a trade-off between the waiting cost of customers and the holding cost of finished products has to be obtained. If the former is very high compared to the latter, always a positive reorder level (that too pretty high) is called for, whereas when the holding cost of finished products is very high in comparison with the cost towards the waiting of customers, the reorder level may tend towards zero. The numerical illustrations are suggestive of these observations.

In this section, we show numerically that it is optimal to place replenishment order before the inventory level drops to zero whenever the waiting cost of customers is very high compared to the holding cost of processed item. Since analytical expressions are not available for the system state probabilities, it is difficult to give a formal proof for the above statement. Neverthless, it can be intuitively shown to hold. For when the customer waiting cost is very high in comparison with the holding cost of processed items, a heavy expenditure is incurred for the former in the absence of processed items. If processed item is available, the demand is immediately met with the result that the waiting cost of customer is completely avoided. This is brought out through Tables 4.4, 4.5, and 4.6. Note that the optimal values of $s$ in these cases are 9, 3, and 1, respectively. In these tables, the service rates are given the values 2.5, 3.0, and 3.5, respectively, and all other parameters are kept fixed. In arriving at the results given in Tables 4.7, 4.8, and 4.9, we constructed a profit function

$$
\widehat{F}(k)=\pi_{0 S k}\left(k \cdot h_{1}^{\prime}+(S-k) h_{2}^{\prime}-\lambda h_{2}\right) .
$$

This is to numerically establish that for a given inventory level, say $S$, there is a corresponding optimal value for the number of processed items to be kept in the inventory. Tables 4.7, 4.8, and 4.9 show that the optimal values of this are 9, 3, and 1, respectively, with service rates $2.5,3.0$, and 3.5 .

We introduce the following cost function. Let the costs associated with the system operation be as follows.

Fixed ordering cost $=K$, procurement cost $=c$ per unit item, holding cost of customers $=h_{2}$ per unit/time, holding cost of processed items $=h_{1}^{\prime}$ per unit/time, and holding cost of unprocessed items $=h_{2}^{\prime}$ per unit/time. 
Table 4.1. $\mu=2.1$.

\begin{tabular}{l|cccccccccc}
\hline$S$ & 12 & 16 & 20 & 24 & 25 & 26 & 27 & 28 & 30 \\
\hline $\begin{array}{l}\text { Average } \\
\text { queue size }\end{array}$ & 11.203 & 10.784 & 10.495 & 10.264 & 10.212 & 10.162 & 10.113 & 10.067 & 9.978 \\
\hline $\begin{array}{l}\text { Average } \\
\text { inventory } \\
\text { held }\end{array}$ & 11.207 & 13.169 & 15.130 & 17.091 & 17.582 & 18.072 & 18.563 & 19.053 & 20.034 \\
\hline $\begin{array}{l}\text { Average } \\
\text { number of } \\
\text { processed } \\
\text { items }\end{array}$ & 2.756 & 3.138 & 3.435 & 3.694 & 3.754 & 3.814 & 3.872 & 3.930 & 4.041 \\
\hline $\begin{array}{l}\text { P (inventory } \\
\text { contains only } \\
\text { processed } \\
\text { items) }\end{array}$ & 0.0464 & 0.0465 & 0.0465 & 0.0466 & 0.0466 & 0.0466 & 0.0466 & 0.0466 & 0.0466 \\
\hline $\begin{array}{l}\text { Average } \\
\text { waiting time } \\
\text { of a customer } \\
\text { in system }\end{array}$ & 5.602 & 5.393 & 5.248 & 5.132 & 5.106 & 5.081 & 5.057 & 5.034 & 4.989 \\
\hline \begin{tabular}{l} 
Expected cost \\
\hline
\end{tabular} & 1437.68 & 1122.88 & 1071.40 & 1058.70 & 1058.04 & 1058.01 & 1058.51 & 1059.47 & 1062.47 \\
\hline
\end{tabular}

We analyse the following cost function:

$$
\begin{aligned}
F(s, S)= & (K+c(S-s))\left(\lambda \sum_{k=1}^{s+1} \pi_{0, s+1, k}+\mu \sum_{i=1}^{\infty} \pi_{i, s+1,0}\right)+h_{1}^{\prime} \sum_{j=s+1}^{S} \sum_{k=1}^{j} k \pi_{0 j k} \\
& +h_{2}^{\prime}\left(\sum_{i=0}^{\infty} \sum_{j=s+1}^{S} \sum_{k=0}^{j} j \pi_{i j k}-\sum_{j=s+1}^{S} \sum_{k=1}^{j} k \pi_{0 j k}\right)+h_{2} \pi_{1}(I-R)^{-2} \mathbf{e} .
\end{aligned}
$$

The objective is to minimize this cost. Since analytical expressions are not available for the system state probabilities, we resort to numerical procedure. Tables 4.1, 4.2, and 4.3 , respectively, show the effect of the maximum inventory level on the system running cost when service rates are $2.1,2.5$, and 3 , respectively, with $\lambda$ fixed at 2 . The values of all other parameters are also kept fixed. Tables 4.4, 4.5, and 4.6 indicate the effect of the replenishment level on the system running cost and other system parameters, with service rates varying as $2.5,3.0$, and 3.5 , respectively. In all these tables, we notice that the cost first shows a decreasing trend, reaches a minimum, and then climbs up.

Effect of $S$ on the expected system cost. Here we take $\lambda=2.0, s=10, K=500, C=100$, $h_{1}^{\prime}=50, h_{2}^{\prime}=10, h_{2}=50$.

Tables 4.4 to 4.6 show effect of replenishment level $s$ on the expected system cost $\lambda=$ 2.0, $S=20, K=50.0, c=20.0, h_{1}^{\prime}=15.0, h_{2}^{\prime}=10.0, h_{2}=200.0$. 
Table 4.2. $\mu=2.5$.

\begin{tabular}{l|cccccccccc}
\hline$S$ & 12 & 14 & 16 & 18 & 19 & 20 & 21 & 22 & 25 \\
\hline $\begin{array}{l}\text { Average } \\
\text { queue size }\end{array}$ & 0.317 & 0.288 & 0.266 & 0.249 & 0.242 & 0.235 & 0.229 & 0.223 & 0.208 \\
\hline $\begin{array}{l}\text { Average } \\
\text { inventory } \\
\text { held }\end{array}$ & 11.354 & 12.356 & 13.356 & 14.355 & 14.854 & 15.354 & 15.853 & 16.353 & 17.850 \\
\hline $\begin{array}{l}\text { Average } \\
\text { number of } \\
\text { processed } \\
\text { items }\end{array}$ & 7.541 & 8.022 & 8.429 & 8.769 & 8.968 & 9.136 & 9.298 & 9.457 & 9.913 \\
\hline $\begin{array}{l}\text { P (inventory } \\
\text { contains only } \\
\text { processed } \\
\text { items) }\end{array}$ & 0.1975 & 0.1977 & 0.1979 & 0.1980 & 0.1981 & 0.1981 & 0.1982 & 0.1982 & 0.1983 \\
\hline $\begin{array}{l}\text { Average waiting } \\
\text { time of a } \\
\text { customer in } \\
\text { system }\end{array}$ & 0.1587 & 0.1439 & 0.1332 & 0.1247 & 0.1210 & 0.1176 & 0.1145 & 0.1116 & 0.1039 \\
\hline \begin{tabular}{l} 
Expected cost \\
\hline
\end{tabular} & 944.04 & 746.21 & 694.26 & 677.72 & 675.25 & 675.06 & 676.56 & 679.32 & 692.58 \\
\hline
\end{tabular}

Table 4.3. $\mu=3.0$.

\begin{tabular}{l|cccccccccc}
\hline$S$ & 12 & 14 & 16 & 18 & 19 & 20 & 21 & 22 & 25 \\
\hline $\begin{array}{l}\text { Average } \\
\text { queue size }\end{array}$ & 0.020 & 0.017 & 0.015 & 0.014 & 0.013 & 0.012 & 0.012 & 0.011 & 0.0096 \\
\hline $\begin{array}{l}\text { Average } \\
\text { inventory } \\
\text { held }\end{array}$ & 11.474 & 12.476 & 13.478 & 13.978 & 14.478 & 14.979 & 15.479 & 16.480 & 17.981 \\
\hline $\begin{array}{l}\text { Average } \\
\text { number of } \\
\text { processed } \\
\text { items }\end{array}$ & 9.333 & 9.889 & 10.374 & 10.602 & 10.822 & 11.036 & 11.246 & 11.653 & 12.241 \\
\hline $\begin{array}{l}\text { P (inventory } \\
\text { contains only } \\
\text { processed } \\
\text { items) }\end{array}$ & 0.3326 & 0.3327 & 0.3328 & 0.3328 & 0.3328 & 0.3329 & 0.3329 & 0.3329 & 0.3330 \\
\hline $\begin{array}{l}\text { Average } \\
\text { waiting time } \\
\begin{array}{l}\text { of a customer } \\
\text { in system }\end{array}\end{array}$ & 0.010 & 0.008 & 0.007 & 0.007 & 0.0066 & 0.0063 & 0.006 & 0.0054 & 0.0048 \\
\hline \begin{tabular}{l} 
Expected cost \\
\hline
\end{tabular} & 879.46 & 713.31 & 677.01 & 672.11 & 671.50 & 673.73 & 677.92 & 690.23 & 714.49 \\
\hline
\end{tabular}


Table 4.4. $\mu=2.5$.

\begin{tabular}{l|cccccccccc}
\hline$S$ & 0 & 3 & 5 & 8 & 9 & 10 & 11 & 12 & 15 \\
\hline $\begin{array}{l}\text { Average } \\
\text { queue size }\end{array}$ & 1.640 & 0.920 & 0.623 & 0.346 & 0.285 & 0.235 & 0.193 & 0.160 & 0.091 \\
\hline $\begin{array}{l}\text { Average } \\
\text { inventory } \\
\text { held }\end{array}$ & 9.971 & 11.566 & 12.671 & 14.298 & 14.828 & 15.353 & 15.875 & 16.394 & 17.934 \\
\hline $\begin{array}{l}\text { Average } \\
\text { number of } \\
\text { processed }\end{array}$ & 2.123 & 3.892 & 5.290 & 7.562 & 8.345 & 9.135 & 9.929 & 10.724 & 13.087 \\
items & & & & & & & & & \\
\hline $\begin{array}{l}\text { P (inventory } \\
\text { contains only } \\
\text { processed } \\
\text { items) }\end{array}$ & 0.1869 & 0.1926 & 0.195 & 0.1972 & 0.1977 & 0.1981 & 0.1984 & 0.1987 & 0.1992 \\
\hline $\begin{array}{l}\text { Average waiting } \\
\text { time of a } \\
\text { customer in } \\
\text { system }\end{array}$ & 0.820 & 0.460 & 0.311 & 0.173 & 0.142 & 0.117 & 0.097 & 0.080 & 0.045 \\
\hline \begin{tabular}{l} 
Expected cost \\
\hline
\end{tabular} & 454.52 & 337.31 & 297.22 & 271.94 & 269.93 & 270.31 & 272.73 & 276.89 & 298.95 \\
\hline
\end{tabular}

Table 4.5. $\mu=3.0$.

\begin{tabular}{l|cccccccccc}
\hline$S$ & 0 & 1 & 2 & 3 & 4 & 5 & 6 & 10 & 15 \\
\hline $\begin{array}{l}\text { Average } \\
\text { queue size }\end{array}$ & 0.442 & 0.309 & 0.216 & 0.150 & 0.104 & 0.072 & 0.050 & 0.012 & 0.002 \\
\hline $\begin{array}{l}\text { Average } \\
\text { inventory } \\
\text { held }\end{array}$ & 10.128 & 10.691 & 11.253 & 11.808 & 12.355 & 12.893 & 13.423 & 15.479 & 17.995 \\
\hline $\begin{array}{l}\text { Average } \\
\text { number of } \\
\text { processed } \\
\text { items }\end{array}$ & 3.472 & 4.173 & 4.915 & 5.683 & 6.466 & 7.259 & 8.056 & 11.246 & 15.138 \\
\hline $\begin{array}{l}\text { P (inventory } \\
\text { contains only } \\
\text { processed } \\
\text { items) }\end{array}$ & 0.317 & 0.3219 & 0.325 & 0.328 & 0.329 & 0.331 & 0.331 & 0.333 & 0.333 \\
\hline $\begin{array}{l}\text { Average waiting } \\
\text { time of a } \\
\text { customer in } \\
\text { system }\end{array}$ & 0.221 & 0.155 & 0.108 & 0.075 & 0.052 & 0.036 & 0.025 & 0.006 & 0.001 \\
\hline \begin{tabular}{l} 
Expected cost \\
\hline
\end{tabular} & 213.2 & 196.2 & 187.18 & 183.84 & 184.5 & 187.95 & 193.35 & 225.53 & 278.89 \\
\hline
\end{tabular}


12 Utilization of idle time in inventory

Table 4.6. $\mu=3.5$.

\begin{tabular}{l|cccccccccc}
\hline$S$ & 0 & 1 & 2 & 3 & 4 & 5 & 7 & 10 & 15 \\
\hline $\begin{array}{l}\text { Average } \\
\text { queue size }\end{array}$ & 0.183 & 0.111 & 0.067 & 0.04 & 0.024 & 0.014 & 0.005 & 0.001 & 0.0001 \\
\hline $\begin{array}{l}\text { Average } \\
\text { inventory } \\
\text { held }\end{array}$ & 10.250 & 10.825 & 11.38 & 11.918 & 12.45 & 12.965 & 13.986 & 15.497 & 17.999 \\
\hline $\begin{array}{l}\text { Average } \\
\text { number of } \\
\text { processed } \\
\text { items }\end{array}$ & 4.452 & 5.2 & 5.965 & 6.738 & 7.513 & 8.288 & 9.833 & 12.133 & 15.88 \\
\hline $\begin{array}{l}\text { P (inventory } \\
\text { contains only } \\
\text { processed } \\
\text { items) }\end{array}$ & 0.414 & 0.420 & 0.423 & 0.425 & 0.427 & 0.427 & 0.428 & 0.428 & 0.429 \\
\hline $\begin{array}{l}\text { Average waiting } \\
\text { time of a } \\
\text { customer in } \\
\text { system }\end{array}$ & 0.092 & 0.055 & 0.033 & 0.020 & 0.012 & 0.007 & 0.003 & 0.0006 & 0.00005 \\
\hline \begin{tabular}{l} 
Expected cost \\
\hline
\end{tabular} & 163.81 & 158.96 & 159.71 & 163.87 & 170.09 & 177.55 & 194.48 & 222.32 & 274.7 \\
\hline
\end{tabular}

Table 4.7. $\mu=2.5, s=9$.

\begin{tabular}{l|ccccccccc}
\hline$k$ & 1 & 4 & 7 & 8 & 9 & 10 & 11 & 15 & 19 \\
\hline$\widehat{F}(k)$ & 0.32 & 0.574 & 0.807 & 0.844 & 3.825 & 2.056 & 1.104 & 0.091 & 0.007 \\
\hline
\end{tabular}

Table 4.8. $\mu=3.0, s=3$.

\begin{tabular}{l|ccccccccc}
\hline$k$ & 1 & 2 & 3 & 4 & 5 & 8 & 11 & 15 & 19 \\
\hline$F(k)$ & 0.231 & 0.272 & 3.821 & 2.231 & 1.301 & 0.257 & 0.05 & 0.006 & 0.0006 \\
\hline
\end{tabular}

Table 4.9. $\mu=3.5, s=1$.

\begin{tabular}{l|cccccccc}
\hline$k$ & 1 & 2 & 3 & 4 & 7 & 11 & 15 & 19 \\
\hline$F(k)$ & 3.539 & 2.194 & 1.359 & 0.842 & 0.199 & 0.029 & 0.004 & 0.0006 \\
\hline
\end{tabular}

Tables 4.7 to 4.9 provide the profit gained by stacking processed items in the inventory. In these tables, we fix $\lambda=2.0, S=20, K=50.0, c=20.0, h_{1}^{\prime}=15.0, h_{2}^{\prime}=10.0, h_{2}=200.0$, the values of $s$ and $\mu$ alone are varied. 


\section{Conclusion}

In conclusion, we have considered in this paper an effective way to improve the server idle-time utilization. Even though the lead time is assumed to be zero, we notice that when the holding cost of customers is very high, reorder level remains positive. In a followup paper, we study a variant of the present one with demands for processed and unprocessed items. This is especially noticed in food processing industries. In this case, there are some customers requiring negligible service time (as in classical inventory models) and some others requiring positive service time. The need for stacking processed items is also brought out in this paper.

\section{Acknowledgments}

The authors thank the referee for several useful comments and suggestions which helped to improve the presentations considerably. A. Krishnamoorthy and T. G. Deepak acknowledge the support from NBHM (DAE, Government of India) through Research Project 48/5/2003/R\&D II/3269 and Vineetha acknowledges financial support from DST.

\section{References}

[1] O. Berman, E. Kim, and D. G. Shimshack, Deterministic approximations for inventory management at service facilities, IIE Transactions 25 (1993), no. 5, 98-104.

[2] O. Berman and E. Kim, Stochastic models for inventory management at service facilities, Communications in Statistics. Stochastic Models 15 (1999), no. 4, 695-718.

[3] O. Berman and K. P. Sapna, Inventory management at service facilities for systems with arbitrarily distributed service times, Communications in Statistics. Stochastic Models 16 (2000), no. 3-4, 343-360.

[4] E. Naddor, Inventory Systems, John Wiley \& Sons, New York, 1966.

[5] M. F. Neuts, Matrix-Geometric Solutions in Stochastic Models. An Algorithmic Approach, Johns Hopkins Series in the Mathematical Sciences, vol. 2, Johns Hopkins University Press, Maryland, 1981.

A. Krishnamoorthy: Department of Mathematics, Cochin University of Science and Technology, Kochi - 682022, India

E-mail address: ak@cusat.ac.in

T. G. Deepak: Government Polytechnic College, Chelad, Kothamangalam 686681, India E-mail address: drtgdeep@yahoo.co.in

Viswanath C. Narayanan: Department of Mathematics, Government Engineering College, Sreekrishnapuram, Palakkad 679513, India

E-mail address: viswam@cusat.ac.in

K. Vineetha: Department of Statistics, University of Calicut, Calicut University, Kerala 673635, India E-mail address: vineethamoosath@yahoo.com 


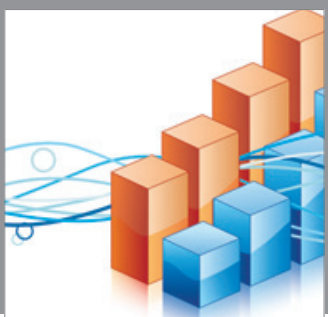

Advances in

Operations Research

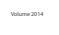

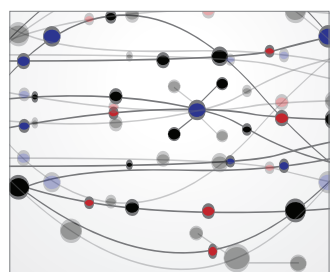

\section{The Scientific} World Journal
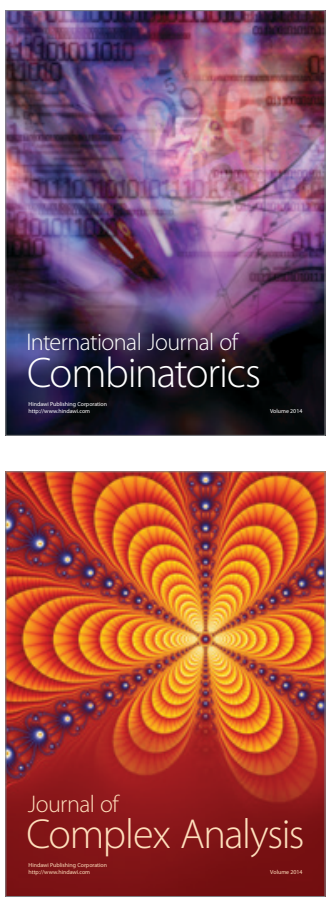

International Journal of

Mathematics and

Mathematical

Sciences
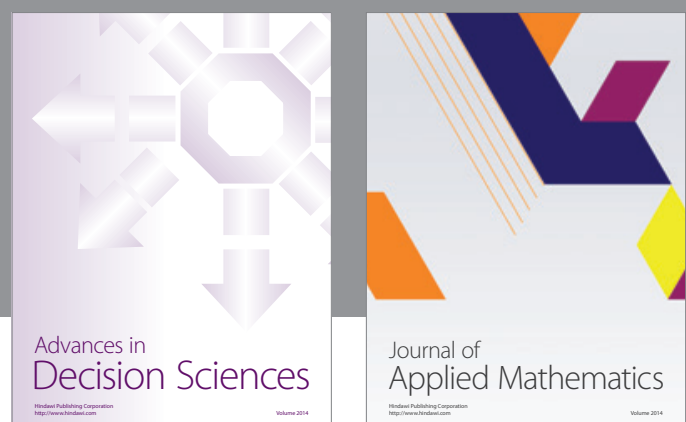

Journal of

Applied Mathematics
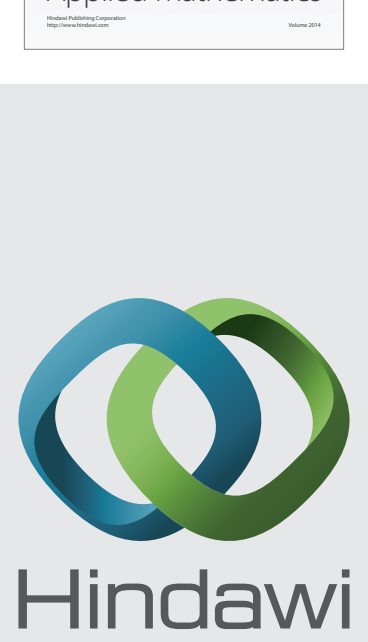

Submit your manuscripts at http://www.hindawi.com
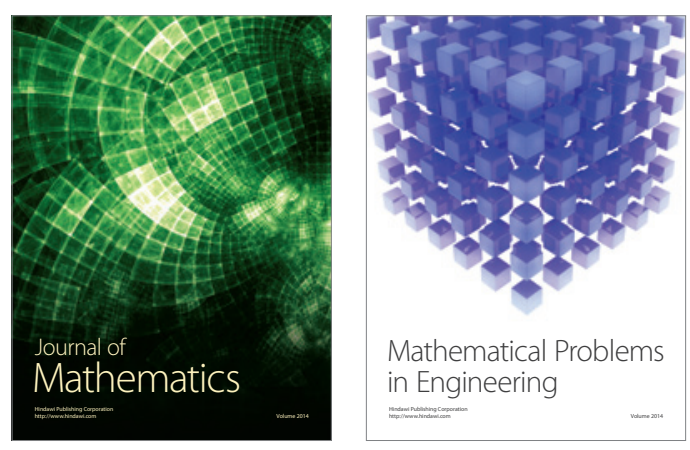

Mathematical Problems in Engineering
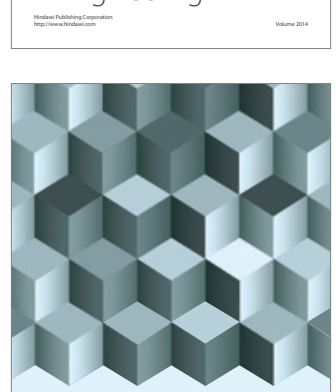

Journal of

Function Spaces
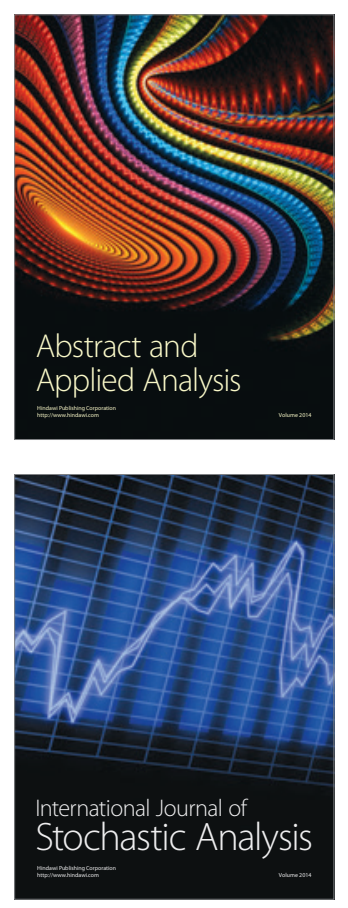

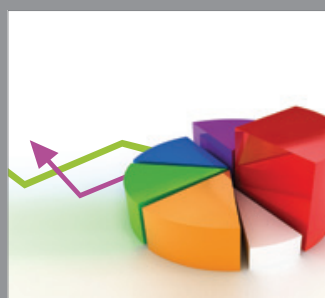

ournal of

Probability and Statistics

Promensencen
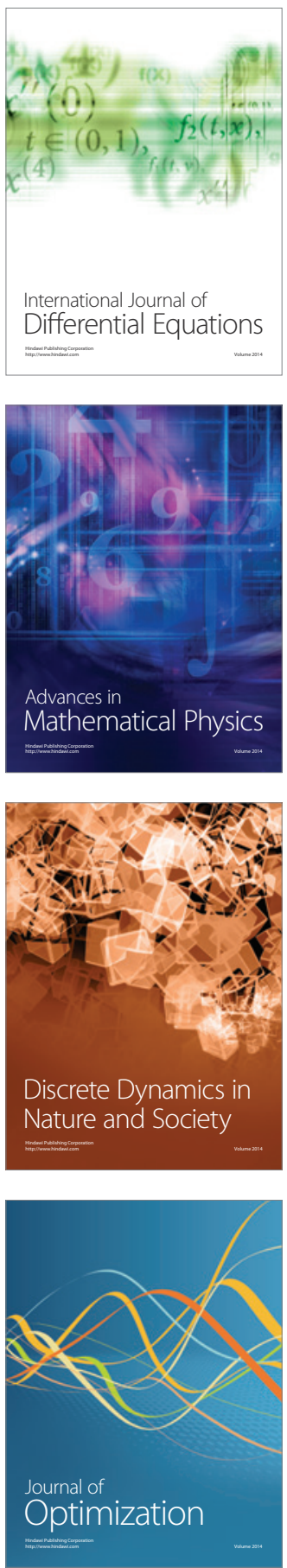